\title{
Prestige latent, variation spatiale et dynamique sociale: le cas des professions intermédiaires
}

\author{
Prikhodkine Alexei \\ Section de linguistique générale \\ Université de Lausanne \\ Alexei.Prikhodkine@unil.ch
}

\begin{abstract}
Dans cet article, nous discutons de la notion de "prestige latent » à la lumière d'une récente recherche centrée sur les attitudes des locuteurs envers le français en usage en Suisse romande. De manière générale, les résultats indiquent que la situation sociolinguistique en Suisse romande est caractérisée par une double dynamique normative : les locuteurs tendent à légitimer tout un pan de variantes lexicales endogènes, tout en dépréciant d'autres et leur préférant les items du français de France. L'enquête a été menée auprès d'un échantillon de 100 individus structuré par plusieurs variables indépendantes. Nous nous centrons en particulier sur quelques résultats inattendus concernant les attitudes des locuteurs envers les variantes endogènes dépréciées (dialectismes et germanismes). L'analyse de variance révèle plusieurs effets d'interaction significatifs entre le sexe et la catégorie socio-professionnelle. Ces effets indiquent un profil évaluatif particulier des hommes appartenant à ce qu'on appelle « professions intermédiaires ». En effet, contrairement aux femmes, ces hommes expriment des attitudes plus favorables envers les variantes dépréciées que les autres membres de la classe moyenne (employés et cadres supérieurs). Cependant, les données n'autorisent pas à interpréter ces résultats en termes de la volonté de ces hommes d'exprimer une qualité désirable de virilité au travers d'éléments linguistiques associés aux hommes de la classe ouvrière. Nous avançons une interprétation alternative liée à la position sociale particulière des membres de la catégorie socio-professionnelle - relativement récente - des professions intermédiaires (intermédiaires de services sociaux et personnels). Nous formulons l'hypothèse que le profil attitudinal de ces hommes peut s'expliquer par un décalage entre leur important capital culturel et leur position socio-économique modeste.
\end{abstract}

\section{Introduction}

En termes de Janet Holmes (2008: 408), le prestige est une notion «glissante», dans la mesure où elle peut référer à des choses différentes. C'est ainsi que le prestige apparent est généralement associé à la variété légitime qui est promue par l'idéologie du standard (Milroy \& Milroy, 1985) relayée par divers organismes faisant autorité (institutions scolaires, médias, grammaires, dictionnaires). Etant donné que le maniement de la variété légitime tend à être présenté par ces instances comme un synonyme de succès professionnel et comme un outil de mobilité sociale ascensionnelle, les variétés linguistiques souffrant de légitimité sont dévaluées et leurs locuteurs marginalisés (Lippi-Green, 1997 ; Heller, 2007). Les pressions sociales sur ces locuteurs peuvent prendre diverses formes, du discours ambiant (au travers des médias écrits et parlés) aux discriminations sur la base du langage (par ex., Purnell \& al., 1999 ; Roy, 2003 ; Archibald, 2009). Dans la mesure où ces pressions font croire que la convergence vers la variété associée au prestige apparent est non seulement souhaitable mais aussi possible, les locuteurs sont en même temps encouragés à se départir de traces linguistiques stigmatisées. A ce propos, l'étude de Provost \& al. (2003) menée en Belgique francophone montre très bien comment une évocation du stéréotype négatif attaché à l'appartenance régionale des sujets parlants peut faire diminuer de leur part une production d'items lexicaux marqués spatialement et favoriser de sorte une convergence vers la variété de prestige. Dans certains cas, le processus peut mener à une extinction complète de traces régionales dévalorisées (Auer, 2005), comme c'est le cas, par exemple, pour la plus grande partie du Danemark. Kristiansen (2009) note, en effet, qu'aujourd'hui les variétés régionales du danois ne se distinguent de la variété de Copenhague que par quelques traits supra-segmentaux principalement. 
Il est établi en même temps qu'une dynamique négative ne caractérise pas toutes les variantes linguistiques en défaut de prestige. La question de savoir pourquoi on n'observe pas une convergence généralisée vers des usages légitimes - qui bénéficient de tout un appareil de promotion - a été posée dès les débuts de la sociolinguistique (cf. Chambers, 2003). Nombre de travaux indiquent aujourd'hui que le prestige apparent peut être contrebalancé par d'autres valeurs, favorables aux variétés dépréciées (cf. Bauvois, 1997). On note cependant une hiérarchie évaluative entre les deux types de valeurs : la variété dominante est perçue plus favorablement sur la dimension relevant de la prescription, tandis que la variété présentant un déficit de légitimité est décrite plus positivement sur les traits relatifs à la sociabilité ou à la loyauté (Ryan \& al., 1982 ; Lafontaine, 1986). La notion d'insécurité linguistique ${ }^{1}$ rend compte, dans son extension psycho-sociale, de la coexistence de plusieurs valeurs: d'une part, une dépréciation de variantes non légitimes, et d'autre part, le recours à des stratégies de compensation en faveur de ces mêmes variantes (Francard, 1993). Afin de rendre la hiérarchie des dimensions évaluatives opératoire au niveau des pratiques linguistiques, les chercheurs belges (Lafontaine, 1986; Francard, 1998) ont proposé d'analyser cette dichotomie au travers du concept de la diglossie (cf. Beniamino, 1997), dans le cadre de laquelle la répartition fonctionnelle des variétés spatiales est rendue possible grâce à la coexistence de plusieurs marchés linguistiques (Bourdieu, 1982). Dans le détail, on note que les auteurs distinguent au moins deux marchés linguistiques: le marché officiel (général) et le marché restreint (local). Le marché linguistique «officiel» est celui où domine le français de France et où sa légitimité est acceptée; le marché linguistique «restreint»est un marché local où s'effectuent des interactions entre les membres de la communauté régionale et où les locuteurs adoptent d'autres valeurs bénéficiant à la variété locale du français.

Ces autres valeurs, favorables à la variété non légitime, ont été appelées «normes cachées » par Labov (1972 : 249). Le qualifiant «cachées» souligne la difficulté d'éliciter ces valeurs dans une interview classique qui tend à reproduire le cadre du marché linguistique officiel. Le terme prestige latent a, lui, été introduit par Trudgill (1972) qui a apporté une première démonstration empirique de l'existence des valeurs tacites. Celles-ci étaient considérées comme un facteur de première importance dans l'explication de la différenciation sexuelle des variables linguistiques à Norwich. Concrètement, Trudgill a découvert un écart significatif entre ce que les locuteurs déclaraient employer et ce qu'ils employaient effectivement, les hommes de la classe moyenne sous-évaluant l'usage des variantes non standard. Cet écart a été interprété comme une attitude favorable - subconsciente ou simplement non avouée - de ces hommes envers les formes linguistiques non légitimes (Trudgill, 1972 : 187). Le chercheur a expliqué le prestige latent attaché aux variantes non standard par des connotations de masculinité qu'elles véhiculaient étant associés aux locuteurs de la classe ouvrière.

Quelles que soient les catégories sociales sous l'analyse, la principale idée derrière la notion de prestige latent est que les locuteurs exprimant plus ou moins consciemment des attitudes positives envers des variantes dépréciées, n'adhèrent pas entièrement aux valeurs sociales dominantes, du moins sur le marché linguistique officiel. Ce constat suppose cependant que l'on s'interroge constamment pourquoi les locuteurs adoptent ce type de comportement. C'est précisément la question que je me suis posée à la découverte de certains résultats inattendus dans le cadre d'une recherche menée en Suisse romande. 


\section{Enquête : indications méthodologiques et résultats généraux}

L'étude d'où sont tirés les résultats analysés dans cet article ${ }^{2}$, visait à examiner la dynamique normative du français en usage en Suisse romande. Celle-ci constitue un espace relativement homogène structuré par l'usage dominant du français. La Suisse romande compose de quatre cantons officiellement monolingues (Genève, Jura, Neuchâtel et Vaud) et des parties francophones de trois cantons bilingues (Berne, Fribourg et Valais). Bien qu'unis par la pratique du même idiome, les Suisses romands ne disposent d'aucune structure politique commune: les principales entités du pouvoir restent les cantons. Ces derniers légifèrent notamment en matière de langues et d'éducation. Il existe, cependant, un certain nombre d'organismes, qui, dépassant les limites cantonales, visent à coordonner les efforts dans tel ou tel domaine. En l'absence d'institutions politiques communes, la mise en place d'organismes tendant à se substituer à ces dernières peut traduire une perception de la Suisse romande comme une région autonome politico-culturellement, perception favorisée, selon Francard (2001 : 44), par une nette domination du français sur son territoire.

Un des aspects les plus importants du paysage linguistique actuel en Suisse romande concerne l'absence de contact du français avec les langues régionales. Cet état de fait clôt un long processus d'unification linguistique, qui a vu le nombre de locuteurs patoisants s'éroder relativement rapidement à l'avantage du français. En effet, tout laisse à supposer que l'usage des patois était encore largement dominant à l'aube du XVII ${ }^{\mathrm{e}}$ siècle dans les limites de la Romandie actuelle ${ }^{3}$ (Knecht, 1985: 145). Tout en étant rapide, la pénétration du français s'est faite graduellement, en devenant d'abord la langue des élites locales, puis en se propageant à une plus large échelle sous l'impact de la Réforme et, plus tard, de l'industrialisation et de l'instruction publique (Knecht, 1996). Au regard de ces dernières impulsions, il n'est pas surprenant de constater que les langues endogènes se sont plus longtemps maintenues dans les régions rurales et catholiques (Knecht, $1985: 143$ ).

Les données ont été récoltées au travers d'entretiens oraux individuels auprès de 100 individus résidant dans les cantons de Vaud, Genève et Fribourg. L'échantillon a été construit selon la technique des quotas ${ }^{4}$ et structuré par plusieurs variables indépendantes, dont le sexe et la catégorie socio-professionnelle (cf. tab. 1).

\begin{tabular}{l|rr|rr|r}
\hline & CS $^{\mathbf{5}}$ & PI & E & M & Total \\
Hommes & 13 & 5 & 11 & 16 & 45 \\
Femmes & 10 & 21 & 18 & 6 & $\mathbf{5 5}$ \\
\hline Total & $\mathbf{2 3}$ & $\mathbf{2 6}$ & $\mathbf{2 9}$ & $\mathbf{2 2}$ & $\mathbf{1 0 0}$ \\
\hline
\end{tabular}

Tableau 1. Echantillon ventilé en fonction des variables sexe et catégorie socio-professionnelle

La variable «catégorie socio-professionnelle» (CSP) est basée sur le premier niveau de la typologie opérée par l'Office fédéral de la statistique (OFS) (Joye \& al., 1995). Cette typologie a l'avantage de proposer une image relativement fine de la structure sociale suisse fondée sur la combinaison de critères relatifs aux compétences organisationnelles et à la formation des individus. La représentativité de la typologie de l'OFS est notamment assurée par une volonté de scinder ce que l'on peut appeler la «classe moyenne», en distinguant plusieurs catégories socio-professionnelles sur la base de la formation, mais aussi de la nature des objets à manier (manuels ou non). Après une adaptation de la typologie à la taille de notre échantillon, l'espace social suisse a été divisé en quatre catégories:

- Cadres supérieurs. En font partie des personnes au bénéfice de la formation supérieure (universitaire ou polytechnique) et celles ayant d'importantes responsabilités d'organisation (par ex., ingénieurs, chercheurs, économistes, avocats).

- Professions intermédiaires. Cette catégorie inclut des personnes généralement en possession d'un diplôme d'une haute école spécialisée et de ressources d'organisation à un degré moindre que les cadres (par ex., assistants sociaux, informaticiens, ergothérapeutes, éducateurs spécialisés). 
- Employés. Font partie de cette catégorie les personnes exerçant une profession qualifiée, demandant une formation professionnelle non universitaire et opérant plutôt sur des documents que sur des objets précis (par ex., comptables, employés de banque et d'administration).

- Manuels. Les personnes qui s'y rangent opèrent plutôt sur des objets, avec ou sans formation professionnelle (par ex., menuisiers, ouvriers de chantier, ferblantiers).

Tous les membres de l'échantillon ont été interviewés selon le même protocole, qui combinait plusieurs techniques relevant des approches directe et indirecte d'investigation des attitudes linguistiques. $\mathrm{Vu}$ la place prépondérante, en termes de nombre d'unités, qu'occupe le lexique parmi les particularités linguistiques du français parlé en Romandie (Knecht, 1996 ; Francard, 1998), les variantes lexicales ont servi de stimuli dans cette étude. Un corpus de quarante-cinq items lexicaux a été sélectionné sur la base du Dictionnaire suisse romand (Thibault \& Knecht, 2004) selon un certain nombre de critères précis (par ex., les éléments lexicaux devaient être répertoriés dans l'ensemble des cantons sous investigation, leurs référents ne devaient pas appartenir à des domaines d'activité spécifiques, etc.). En fonction des sources alimentant le français parlé en Suisse romande (Schüle, 1981), quatre types de régionalismes ont été distingués : dialectismes, germanismes, archaïsmes et innovations. Pour des raisons de faisabilité, le corpus ainsi obtenu a ensuite été subdivisé en deux sous-corpus (cf. l'annexe). Les éléments du premier corpus devaient apparaître dans les premières phases de l'entretien et faire office de base de comparaison pour les items du deuxième corpus.

En termes d'objectifs, nous nous attachions à voir si les locuteurs romands ont intériorisé la conception pluricentrique de la norme (Pöll, 1998) en rendant leur variété linguistique distincte de l'idiome hexagonal et en s'affirmant, en quelque sorte, propriétaires de leur langue. En soulignant les limites des études antérieures ayant conclu à une dépréciation de la variété endogène (L'Eplattenier-Saugy, 1999 ; Singy, 1996, 2004), nous postulions que la situation sociolinguistique en Suisse romande est caractérisée par une double dynamique normative, qui peut s'expliquer par des raisons socio-historiques liées à l'implantation du français dans les limites de la Suisse romande. D'un côté, les locuteurs sont enclins à se départir des variantes résultant d'un contact de langues (dialectismes et germanismes). De l'autre, ces mêmes locuteurs ont tendant à légitimer des éléments endogènes relevant du français, à savoir des archaïsmes (i.e. les termes qui sont toujours en usage en Suisse romande mais plus dans le français de France dans sa version standard) et des innovations (i.e. les items qui résultent de la dynamique propre du français parlé en Romandie).

L'analyse des résultats a confirmé nos hypothèses, en faisant apparaître, sur la plupart des indicateurs, deux classes d'unités endogènes bien distinctes. Brièvement, ces résultats montrent que les traits lexicaux endogènes relevant d'innovations et d'archaïsmes disputent la conformité à la norme prescriptive aux mots du français de France (cf. graph. 1 à titre d'exemple) et bénéficient, en même temps, d'un usage exclusif de la part du plus grand nombre de répondants. La légitimation de ces termes endogènes bénéficie, par ailleurs, d'un large consensus auprès de la population.

Contrairement à ces unités, le prestige apparent ne semble pas être du côté des variantes dialectales et germaniques. En effet, comme le graphique 1 le montre, tous les éléments relevant des dialectismes et des germanismes sont clairement perçus comme moins corrects que les variantes correspondantes du français de France. De plus, l'usage déclaré de ces variantes endogènes se raréfie au fur et à mesure que l'on s'élève dans la hiérarchie sociale. Il est à noter que les résultats attachés au corpus II sont largement convergents à ceux que nous venons de présenter. 


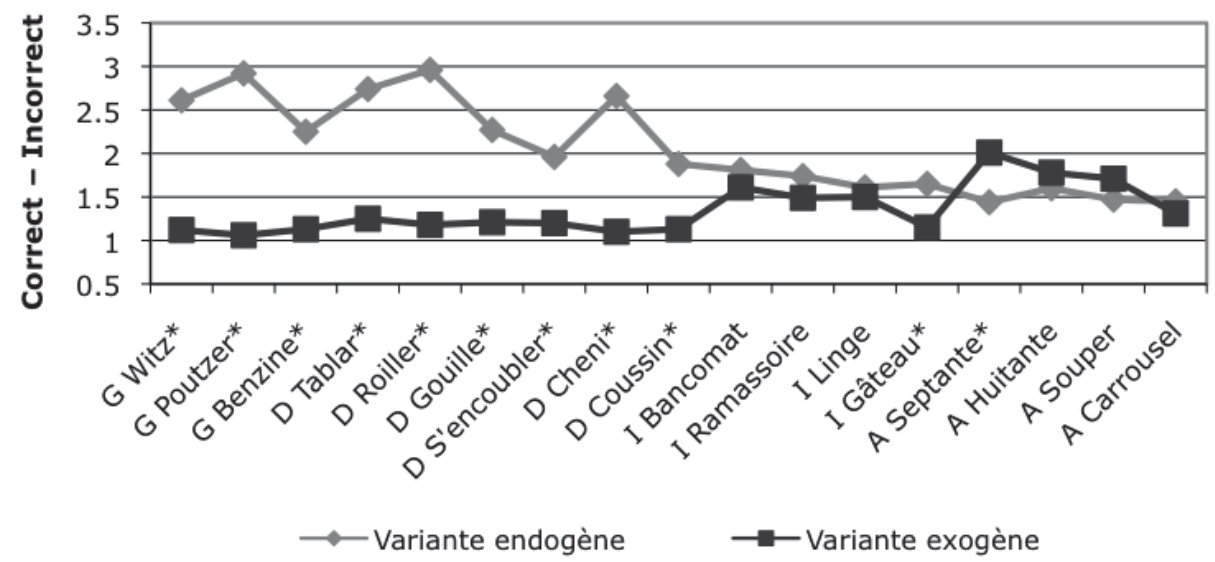

Graphique 1. Evaluation des variables du corpus I sur la dimension prescriptive (test $\mathrm{T}$ pour échantillons appariés ; * $\mathrm{p}<.05)^{6}$

\section{Résultats inattendus ${ }^{7}$}

Pour rendre compte des effets d'interaction entre les variables indépendantes, nous avons effectué des analyses de variance ${ }^{8}$ à plusieurs facteurs. Celles-ci ont indiqué plusieurs effets significatifs, dont l'essentiel concerne les attitudes des locuteurs envers les variantes endogènes dépréciées (dialectismes et germanismes). La particularité de ces résultats est qu'ils font apparaître sur plusieurs indicateurs un pattern similaire d'interaction du genre et de la catégorie socio-professionnelle.

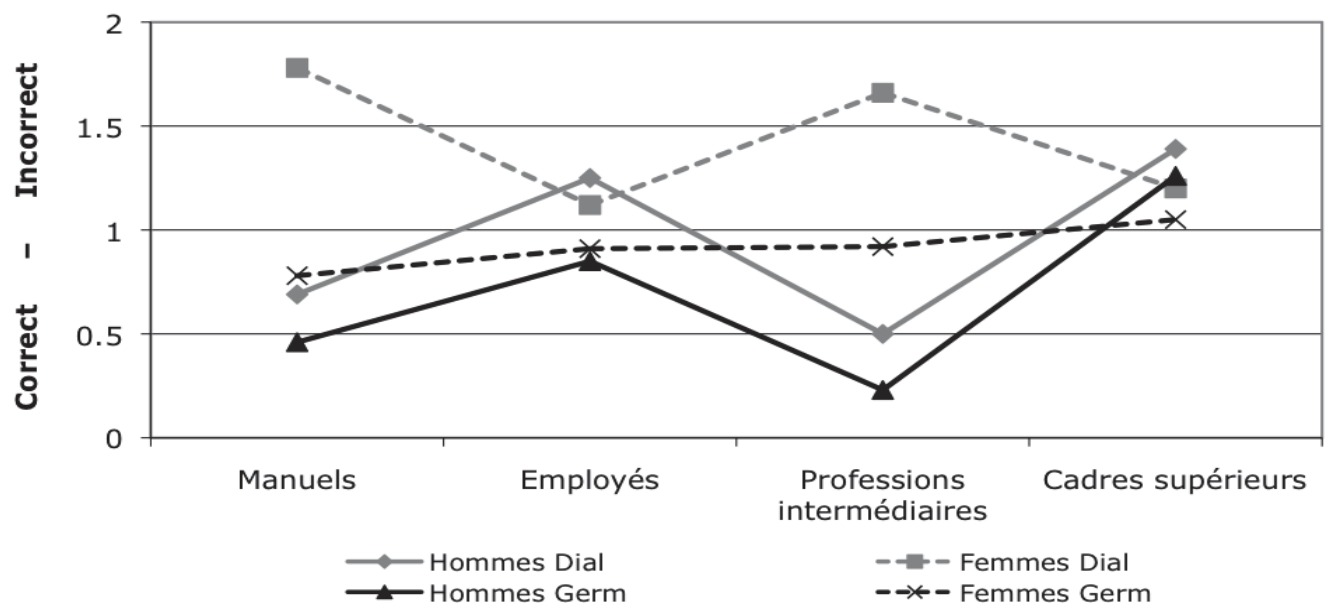

Graphique 2. Evaluation des dialectismes $(F(3)=6.713, p=.000)$ et des germanismes $(F(3)=2.680, p=.052)$ du corpus I sur la dimension prescriptive: résultats d'interaction de la catégorie socio-professionnelle et du genre ${ }^{9}$

Le graphique 2 fait apparaître ce pattern sur la dimension prescriptive. Nous y observons une tendance similaire pour les deux types de régionalismes: les hommes et les femmes des professions intermédiaires ne se rejoignent pas dans leur appréciation des variantes endogènes. Les femmes ont en effet une plus grande propension à déprécier les items régionaux que les hommes. 
Exactement le même schéma évaluatif caractérise les éléments lexicaux du corpus II pour ce qui est de l'écart séparant les hommes et les femmes au sein de la catégorie des professions intermédiaires sur la dimension prescriptive (cf. graph. 3). En effet, si les femmes de cette catégorie socio-professionnelle témoignent de l'attitude la moins tolérante envers les dialectismes et les germanismes, les hommes membres des professions intermédiaires se distinguent des autres informateurs masculins par la perception la moins négative des termes endogènes.

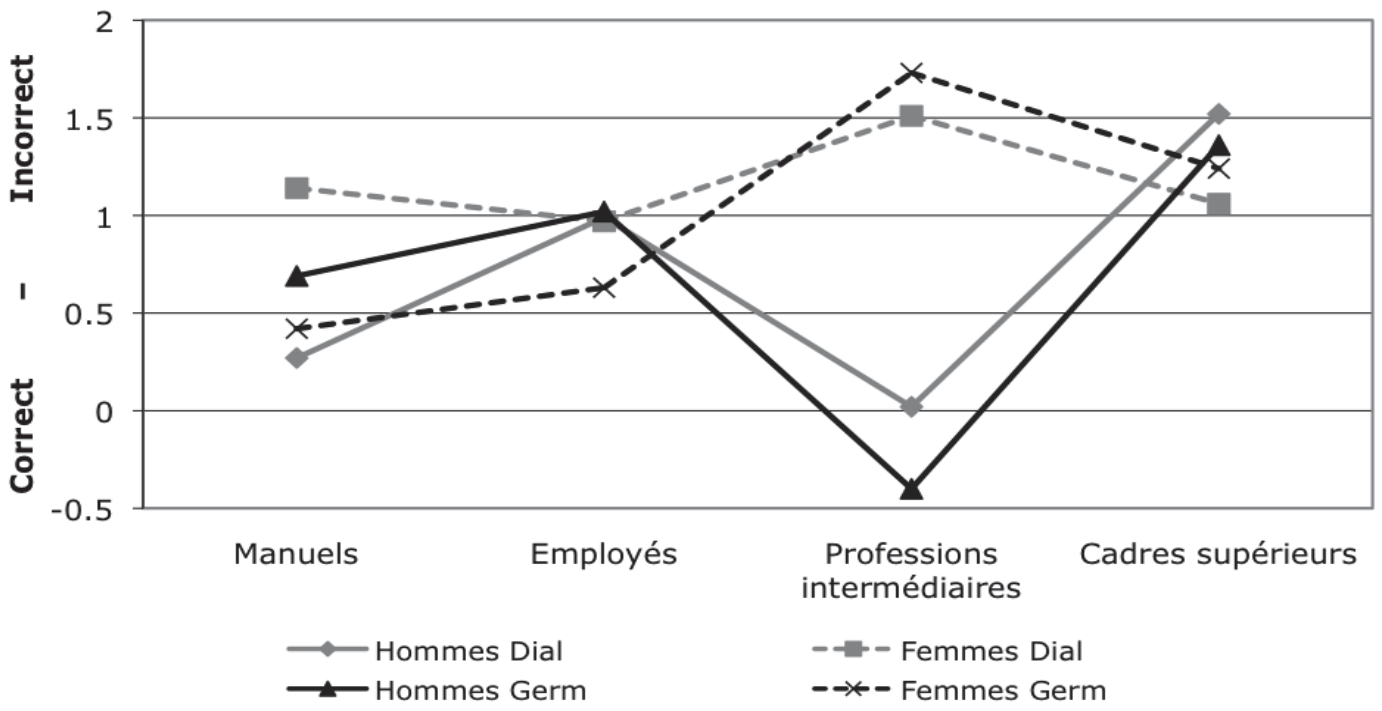

Graphique 3. Evaluation des dialectismes $(\mathrm{F}(3)=4.181, \mathrm{p}=.008)$ et des germanismes $(\mathrm{F}(3)=5.513, \mathrm{p}=.002)$ du corpus II sur la dimension prescriptive: résultats d'interaction de la catégorie socio-professionnelle et du genre

La dimension prescriptive faisait partie de la phase d'évaluation de l'entretien, dans le cadre de laquelle l'ensemble des personnes interrogées ont été invitées à qualifier chaque paire de variantes lexicales sur cinq attributs de comparaison, se présentant sous forme de différenciateurs sémantiques: «joli-laid» relèvent de la dimension esthétique, «plaisant-déplaisant» renvoient à la dimension affective, «élégantlourd» fondent la dimension «raffinement», «correct-incorrect» relèvent de la dimension prescriptive et «chaleureux-froid» renvoient à la dimension «sociabilité». Cette dernière - que l'on peut désigner aussi «loyauté» - représente, avec l'attribut prescriptif, les deux grands axes sur lesquels les attitudes tendent généralement à varier (Ryan \& al., 1982). L'interaction entre ces dimensions s'inscrit souvent dans le cadre du modèle diglossique, lorsque les traits régionaux sont perçus comme inférieurs quant à leur prestige apparent mais supérieurs en ce qui concerne la valeur de sociabilité qu'ils véhiculent (par ex., Francard, 1993). Un tel profil d'interaction de deux dimensions caractérise, dans une certaine mesure, nos résultats attachées aux variantes dialectales et germaniques. En effet, les premières, plus que les secondes, peuvent se prévaloir d'un certain capital de sympathie pour nos informateurs. Cette dimension de sociabilité voit également se creuser des écarts entre les franges de notre échantillon, les hommes des professions intermédiaires insistant beaucoup plus que les autres sur le caractère attachant des régionalismes (cf. graph. 4). Il est à noter que le comportement singulier des membres masculins de cette catégorie socio-professionnelle s'observe dans l'évaluation des items des deux corpus.

Afin de ne pas multiplier les résultats mettant au jour ce pattern perceptif, nous allons nous limiter à dresser un constat général, relatif aux parties de l'entretien où l'on observe ce profil particulier. En effet, celui-ci a apparu principalement lors de la phase d'évaluation (i.e. phase écrite), lorsque les locuteurs devaient remplir des grilles d'évaluation sans se prononcer à haute voix sur leurs préférences envers tel 
ou tel terme. Fait intéressant, le profil singulier des hommes des professions intermédiaires est moins apparent dans les réponses exprimées lors de la partie semi-directive de l'interview, voire absent lorsque les locuteurs devaient rapporter leur propre usage des variantes régionales. Dans ces conditions, nous sommes tenté de supposer que les valeurs positives attachées par ces hommes aux items endogènes par ailleurs généralement dépréciés ne sont pas exprimées ouvertement en présence d'un enquêteur et relèvent de ce que Labov a appelé des normes cachées (cf. § 1).

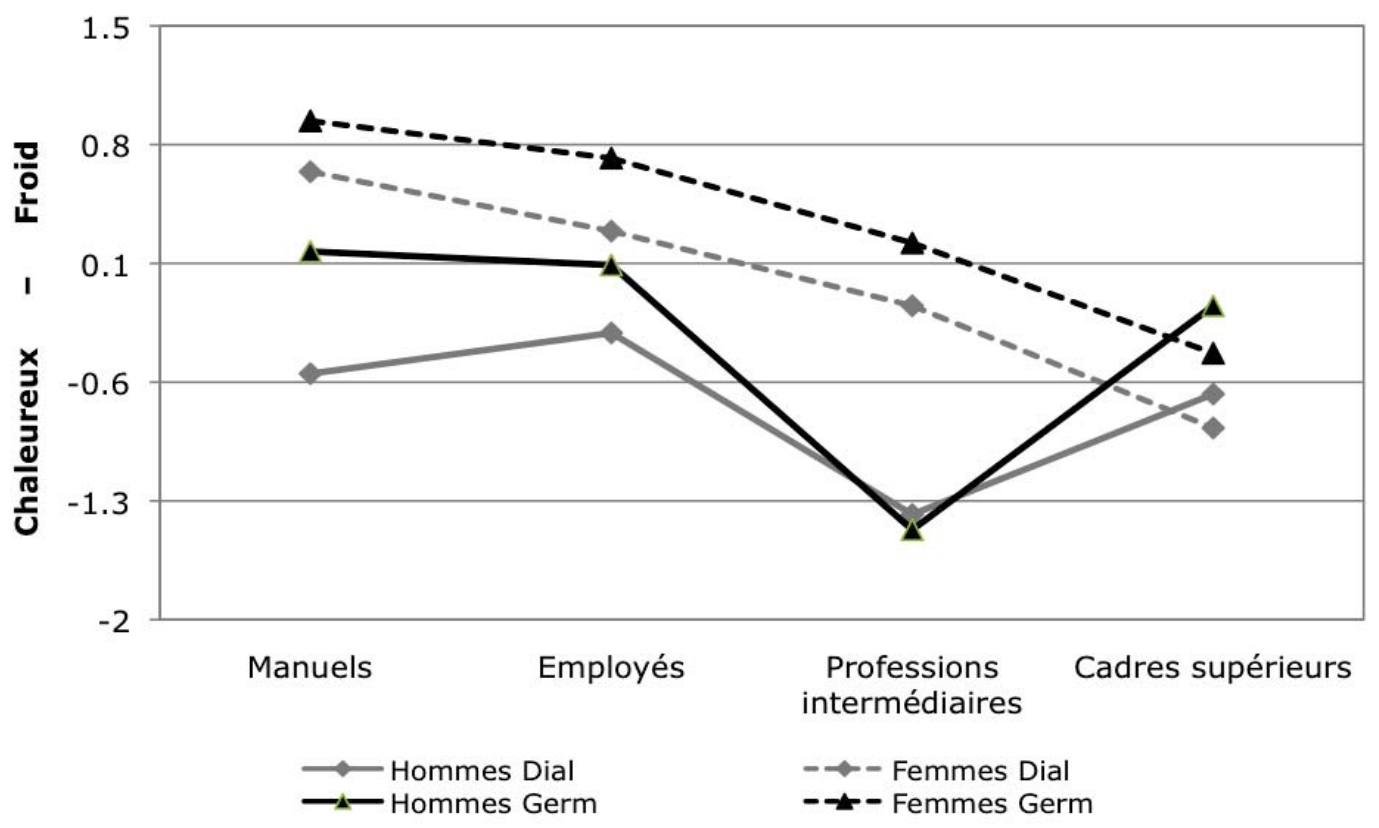

Graphique 4. Evaluation des dialectismes $(F(3)=2.955, p=.035)$, des germanismes $(F(3)=3.462, p=.020)$ du corpus I sur la dimension «sociabilité»: résultats d'interaction de la catégorie socio-professionnelle et du genre

Avant d'entreprendre une interprétation de ces résultats, il convient de s'interroger sur la portée des réponses exprimées par un nombre restreint d'individus qui forment la catégorie des hommes des professions intermédiaires. Au moins trois arguments militent en faveur de la validité des résultats. D'abord, la rigueur de la construction de l'échantillon et une définition pertinente de la structure sociale de la population suisse soutiennent la généralisation des données (Joye \& al., 1995). Ensuite, l'analyse de variance effectuée sur celles-ci tient compte de la taille des groupes et le fait qu'un effet significatif est observé dans un échantillon aux groupes relativement petits peut témoigner de la robustesse de l'analyse statistique (Norusis, 2008). Enfin, le troisième argument concerne la validité des données en sciences sociales. Nous savons en effet (Grawitz, 1984) que pour mesurer des phénomènes sociaux, nous devons, d'une part, rendre les concepts opératoires en les traduisant en indicateurs directement observables, et d'autre part, multiplier ces derniers afin de s'assurer que nous mesurons les manifestations du même phénomène. En ce sens, les résultats exposés dans les pages qui précèdent possèdent une certaine validité, dans la mesure où le même pattern évaluatif ressort sur plusieurs indicateurs. 


\section{Discussion}

Il s'agit maintenant d'avancer une explication à cette tendance des membres masculins de la catégorie socio-professionnelle «professions intermédiaires » à exprimer des attitudes favorables aux variantes endogènes dépréciées. Il convient d'abord de se demander s'il est possible d'interpréter ce phénomène dans le sillage de Trudgill (1972), c'est-à-dire par la volonté de ces hommes d'exprimer une qualité désirable de virilité au travers de termes associés à leurs utilisateurs privilégiés, à savoir les hommes dont l'appartenance socio-professionnelle est fondée sur le recours à la force physique (Prikhodkine \& Singy, 2006). Plusieurs indicateurs de notre recherche visaient précisément à établir dans quelle mesure les items lexicaux régionaux possédaient de telles connotations aux yeux de nos informateurs. Parmi ces indicateurs, deux mesuraient le phénomène de manière directe (les questions portaient explicitement sur les qualités de virilité et de charme féminin) et un - de façon indirecte (la question portait sur la lourdeur perçue des unités lexicales). Les réponses à ces questions ne permettent pas de retenir cette explication. En effet, contrairement aux travailleurs manuels, les membres masculins des professions intermédiaires ne sont pas portés à attacher de connotations de masculinité à ces régionalismes.

Pour interpréter cette tendance à la valorisation des usages linguistiques par ailleurs stigmatisés, il n'est pas déraisonnable, à notre avis, d'invoquer la position sociale particulière des membres de la catégorie socio-professionnelle des professions intermédiaires. Une des spécificités de cette catégorie est d'être composée, en grande partie, de professions «réinvesties» - d'intermédiaires de services sociaux et personnels, dont les effectifs sont en forte croissance ces dernières années, avec le développement notamment de formations professionnelles supérieures (hautes écoles spécialisées ou HES) se distinguant aussi bien de la formation universitaire que de celle de l'apprentissage. Une autre particularité des membres de ces professions est de posséder un important capital culturel et linguistique - une formation professionnelle poussée, qui, souvent marquée par son orientation sociale, se focalise sur l'acquisition de connaissances et sur la communication (Joye \& al., 1995).

Les spécificités de cette catégorie socio-professionnelle tendent à coïncider avec les traits que Bourdieu attribue à la «petite bourgeoisie nouvelle» (1979). Pour le sociologue français, cette frange de la classe moyenne serait sensible au décalage entre ses importantes ressources culturelles et la position socioéconomique qu'elle occupe effectivement. Une des conséquences de cette situation en porte-à-faux serait la volonté, manifeste ou latente, de ses membres de se distinguer par une remise en question de la hiérarchie sociale et par une valorisation de pratiques stigmatisées (Bourdieu, 1979).

Si cette hypothèse peut rendre compte de la tendance des membres des professions intermédiaires à attacher un certain prestige latent aux formes lexicales dépourvues de prestige apparent, elle n'explique pas des différences observées entre les profils attitudinaux des hommes et des femmes. Pourquoi, en effet, cette propension à légitimer les usages linguistiques «déclassés» ne caractérise-t-elle que les hommes des professions intermédiaires? Pour essayer de comprendre ces disparités, il nous semble pertinent d'invoquer la persistance des discriminations salariales entre hommes et femmes possédant les mêmes qualifications. Un rapport de l'Office fédéral de la statistique (OFS, 2008) fait état de telles discriminations tant dans le secteur privé que public. On apprend qu'en 2006 les disparités salariales atteignent, en moyenne, $12.9 \%$ dans le secteur public fédéral et $18.8 \%$ dans le secteur public cantonal, cette différance s'élevant à un niveau semblable à celui du secteur privé (OFS, 2008: 22-23). Dans ces conditions, pour expliquer les écarts entre hommes et femmes dans notre étude, nous pouvons invoquer l'hypothèse de type sociologique en lien avec la position socio-économique inégalitaire des deux sexes: les femmes, bénéficiant d'un pouvoir économique souvent plus faible, ne peuvent signaler leur statut et leur position sociale qu'en recourant aux marques symboliques de pouvoir que constituent les pratiques linguistiques des groupes sociaux dominants (cf. Pillon, 1997). En d'autres termes, on est fondé à supposer qu'afin d'établir une certaine égalité sur le plan socio-professionnel, les femmes sont plus portées à vouloir confirmer leur capital culturel au travers de l'emploi des variantes légitimes, au contraire des hommes. 
Aucune étude, à notre connaissance, n'a scruté les comportements et les représentations de cette nouvelle catégorie socio-professionnelle constituée en grande partie d'intermédiaires des services sociaux et personnels, dont les effectifs sont en constante augmentation. Aussi, il serait utile d'entreprendre une nouvelle recherche auprès d'un échantillon plus important de représentants de ces professions, interrogeant tant les attitudes que les pratiques linguistiques de ces derniers, pour tester l'hypothèse selon laquelle la volonté des membres des professions intermédiaires de se distinguer par une remise en question de la hiérarchie sociale en conférant un prestige latent aux pratiques stigmatisées peut s'expliquer par leur position sociale particulière qui consiste en un décalage entre leur capital culturel et linguistique (une formation poussée, souvent une haute école spécialisée) qui les apparente aux cadres supérieurs et leur position socio-économique qui les rapproche des employés. Enfin, s'agissant du degré de confirmation de cette hypothèse, il apparaît important, dans l'avenir, d'accroître la quantité de données empiriques, en étendant l'investigation à d'autres niveaux de la structure linguistique.

\section{Références bibliographiques}

Archibald, J. (2009). Qu'est-ce que le linguicisme?. In Archibald, J. et Galligani, S. (dir.), Langue(s) et immigration(s) : société, école, travail. Paris : L'Harmattan, 33-48.

Auer, P. (2005). Europe's sociolinguistic unity, or: A typology of European dialect/standard constellations. In Delbecque, N., Van Der Auwera, J. et Geeraerts, D. (éd.), Perspectives on Variation: sociolinguistic, historical, comparative. Berlin : De Gruyter, 7-42.

Bauvois, C. (1997). Prestige apparent vs prestige latent. In Moreau, M.-L. (dir.), Sociolinguistique. Concepts de base. Sprimont : Mardaga, 235-236.

Beniamino, M. (1997). Diglossie. In Moreau, M.-L. (dir.), Sociolinguistique. Concepts de base. Sprimont : Mardaga, 125-129.

Bourdieu, P. (1982). Ce que parler veut dire. Paris : Fayard.

Bourdieu, P. (1979). La distinction. Paris : Editions de Minuit.

Chambers, J. K. (2003). Sociolinguistic theory. Oxford : Blackwell, $2^{\text {nd }}$ edition.

Francard, M. (2001). Français de frontière: la Belgique et la Suisse francophones. Présence Francophone, 56, $27-54$.

Francard, M. (1998). Entre pratiques et représentations linguistiques: le lexique des Belges francophones. In Marle, D., Hintze, M.-A. et Parker, G. (ed.), Linguistic Identities and Policies in France and the French-speaking World. London : CILT, 149-159.

Francard, M. (1993). L'insécurité linguistique en communauté française de Belgique. Bruxelles : Service de la langue française.

Grawitz, M. (1984). Méthodes des sciences sociales. Paris : Dalloz.

Heller, M. (éd.) (2007). Bilingualism : A social approach. New-York : Palgrave Macmillan.

Holmes, J. (2008). An introduction to sociolinguistics. Essex : Pearson Education Limited, 3rd edition.

Huot, R. (2003). Méthodes quantitatives pour les sciences humaines. Québec : Les Presses Universitaires de Laval.

Joye, D., Schuler, M. et Meier, U. (1995). La structure sociale de la Suisse: catégories socio-professionnelles. Berne : OFS

Knecht, P. (1996). La Suisse romande: aspects d'un paysage francophone conservateur. In Robillard, D. \& Beniamino, M. (éd.), Le français dans l'espace francophone. Paris: Honoré Champion, vol. 2, 759-770.

Knecht, P. (1985). La Suisse romande. In Schläpfer, R. (dir.), La Suisse aux quatre langues. Genève : Editions Zoé, 125-169.

Kristiansen, T. (2009) The macro level social meanings of late modern Danish accents. Acta Linguistica Hafniensia, 40(1), 167-192.

Labov, W. (1972). Sociolinguistic patterns. Philadelphia : University of Pennsylvania Press. 
Lafontaine, D. (1986). Le parti pris des mots. Normes et attitudes linguistiques. Bruxelles : Mardaga.

L’Eplattenier-Saugy, C. (1999). A perceptual dialect study of French in Switzerland. In Long, D. et Preston, D. R. (éd.), Handbook of Perceptual Dialectology, vol. 2. Amsterdam/Philadelphia : John Benjamins, 351-365.

Lippi-Green, R. (1997). English with an Accent. Language, ideology, and discrimination in the United States. NewYork - London : Routledge.

Milroy, J. et Milroy, L. (1985). Authority in language. Investigating language prescription and standardisation. Londonb: Routledge \& Kegan Paul.

Norušis, M. J. (2008). SPSS 17.0 Statistical Procedures Companion. Prentice Hall.

Office Fédéral De La Statistique (2008). Vers l'égalité entre femmes et hommes. Neuchâtel/Berne : OFS.

Pillon, A. (1997). Sexe. In Moreau, M.-L. (dir.), Sociolinguistique. Concepts de base. Sprimont : Mardaga, 258-265.

Pöll, B. (1998). Le français ou les français? La difficile naissance de la pluricentricité. Lengas, 43, 163-182.

Prikhodkine, A. (2011). Dynamique normative du français en usage en Suisse romande. Enquête sociolinguistique dans les cantons de Vaud, Genève et Fribourg. Paris : L'Harmattan.

Prikhodkine, A. et Singy, P. (2006). Variétés locales du français et normes cachées. In Lopez Diaz, M. et Montes Lopez, M. (éd.), Perspectives fonctionnelles: emprunts, économie et variation dans les langues. Lugo : Editorial Axac, 287-290.

Provost, V., Yzerbyt, V., Corneille, O., Désert M. et Francard, M. (2003). Stigmatisation sociale et comportements linguistiques : Le lexique menacé. Revue internationale de psychologie sociale, 16 (1), 177-200.

Purnell, Th., Idsardi, W. et Baugh, J. (1999). Perceptual and Phonetic Experiments on American English Dialect Identification. Journal of Language and Social Psychology, 18 (1), 10-30.

Roy, S. (2003). Bilingualism and Standardization in a Canadian Call Center : Challenges for a Linguistic Minority Community. In Bayaley, R. \& Schecter, S. R. (éd.), Language Socialization in Bilingual and Multilingual Societies. Toronto : Multilingual Matters LTD, 269-285.

Ryan, E., Giles, H. et Sebastian, R. J. (1982). An integrative perspective for the study of attitudes toward language variation. In Ryan, E. et Giles, H. (éd.), Attitudes towards Language Variation. London : Edward Arnold, 1-19.

Schüle, E. (1981). Le français régional de Suisse». Actes du colloque «Les français régionaux», Québec, 21-25 octobre 1979. Québec : Editeur officiel du Québec, 181-193.

Singy, P., Mottaz Bara, A., Amstalde, M., Prikhodkine, A. et Jufer, N. (2004). Identités de genre, identités de classe et insécurité linguistique. Berne : Peter Lang.

Singy, P. (1996). L'image du français en Suisse romande. Paris : L'Harmattan.

Thibault, A. et Knecht, P. (dir.) (2004). Dictionnaire suisse romand. Nouvelle édition. Genève : Zoé.

Trudgill, P. (1972). Sex, covert prestige, and linguistic change in the urban British English of Norwich. Language and Society, 1, 179-195. 


\section{Annexe}

Corpus I

\begin{tabular}{lll}
\hline Dialectismes & Variantes endogènes & Variantes exogènes \\
& S'encoubler & Trébucher \\
& Gouille & Flaque \\
& Roiller & Pleuvoir à verse \\
& Tablar & Etagère \\
& Cheni & Désordre \\
& Coussin & Oreiller \\
\hline \multirow{2}{*}{ Germanismes } & Witz & Blague \\
& Poutzer & Nettoyer \\
& Benzine & Essence \\
\hline \multirow{2}{*}{ Archaïsmes } & Septante & Soixante-dix \\
& Huitante & Quatre-vingt \\
& Carrousel & Manège \\
& Souper & Dîner \\
\hline Innovations & Bancomat & Distributeur \\
& Ramassoire & Pelle à poussière \\
& Gâteau & Tarte \\
& Linge & Serviette \\
\hline
\end{tabular}




\section{Corpus II}

\begin{tabular}{|c|c|c|}
\hline & Variantes endogènes & Variantes exogènes \\
\hline \multirow[t]{12}{*}{ Dialectismes } & Bourillon & Nombril \\
\hline & Cocoler & Choyer \\
\hline & Déguiller & Tomber \\
\hline & Eclaffer & Ecraser \\
\hline & Goger & Tremper \\
\hline & Planelle & Carreau \\
\hline & Rogneux & Chicaneur \\
\hline & Commerce & Remue-ménage \\
\hline & Grailler & Gratter \\
\hline & Moque & Morve \\
\hline & Patte & Chiffon \\
\hline & Relaver & Faire la vaisselle \\
\hline \multirow[t]{4}{*}{ Germanismes } & Foehn & Sèche-cheveux \\
\hline & Stempel & Tampon \\
\hline & Chablon & Pochoir \\
\hline & Colonne (d'essence) & Pompe (à essence) \\
\hline \multirow[t]{4}{*}{ Archaïsmes } & Costume de bain & Maillot de bain \\
\hline & Etude (d'avocat) & Cabinet (d'avocat) \\
\hline & Malhonnête & Impoli \\
\hline & Pousse-pousse & Poussette \\
\hline \multirow[t]{8}{*}{ Innovations } & Ecole enfantine & Ecole maternelle \\
\hline & Se gaffer & Faire attention \\
\hline & Petits fruits & Fruits rouges \\
\hline & Sous-voie & Passage souterrain \\
\hline & Agencée (cuisine) & Aménagée (cuisine) \\
\hline & Comptoir & Foire \\
\hline & Course & Excursion \\
\hline & Régater & Rivaliser \\
\hline
\end{tabular}


${ }^{1}$ L'expression «insécurité linguistique» apparaît pour la première fois dans les travaux de Labov rendant compte de son enquête sur la stratification sociale des variables phonologiques à New-York (cf. Labov, 1972). L'insécurité linguistique mesurée par Labov à partir de l'écart entre «usage personnel» et «usage correct», se manifeste, au niveau des comportements, notamment par un effort conscient de correction et des réactions fortement négatives envers la façon de parler héritée.

${ }^{2}$ Pour un aperçu complet des dimensions investiguées, cf. Prikhodkine, 2011.

${ }^{3}$ L'espace romand est partagé entre deux zones dialectales : le domaine francoprovençal et la zone d'oïl. Seuls le canton de Jura et deux districts bernois relèvent de cette dernière zone (Knecht, 1996: 760-761).

${ }^{4}$ Le prélèvement de l'échantillon par quotas consiste en un procédé empirique qui reproduit la logique du choix au hasard. S'apparentant à l'échantillon stratifié, il est le résultat du découpage de la population selon certains traits bien précis (Huot, 2003: 15): les quotas des individus présentant des caractéristiques pertinentes pour l'étude doivent être identiques à la distribution de celles-ci au sein de la population définie. En admettant l'interdépendance des variables attachées à l'individu, on postule généralement que l'échantillon par quotas est peu différent de la population en ce qui concerne la distribution des variables non contrôlées (Grawitz, 1984: 577).

${ }^{5}$ Les abréviations relatives aux catégories socio-professionnelles se lisent comme suit: CS - cadres supérieurs; PI personnes exerçant des professions intermédiaires; $\mathrm{E}$ - employés; $\mathrm{M}$ - personnes exerçant des professions manuelles.

${ }^{6}$ Les symboles 'A/I/D/G' devant les items renvoient à la catégorie des variantes endogènes et doivent se lire comme suit: archaïsmes, innovations, dialectalismes, germanismes. Par ailleurs, pour ne pas alourdir la présentation graphique des résultats, la dénomination de chaque variable se présente sous la forme de la variante endogène.

${ }^{7}$ Ces résultats présentent un caractère inattendu dans le sens où ils n'étaient pas anticipés par les termes de nos hypothèses formulées avant l'expérimentation.

${ }^{8}$ L'analyse de variance teste la significativité du lien entre une ou plusieurs variables catégorielles et une variable numérique.

${ }^{9}$ Les scores indiqués représentent la différence entre les moyennes des variantes endogènes et exogènes. Lorsque le score est positif, la moyenne des variantes endogènes est supérieure à celle des éléments exogènes. Le rapport est inversé lorsque le score est négatif. 